\title{
Response letter to editor-surgical revascularisation for childhood moyamoya
}

\author{
Joanne Ng • Dominic Thompson • John P. S. Lumley • \\ Dawn E. Saunders • Vijeya Ganesan
}

Received: 25 July 2012 / Accepted: 27 July 2012 /Published online: 16 August 2012

(C) Springer-Verlag 2012

\section{Dear Editor:}

We are grateful to Dr Scott's kind comments and highlighting an important point. In our table, we combined all reported mortality both operative and long-term deaths as reporting of mortality differs between centres. The Stanford series report operative deaths only [1], whilst Dr. Scott's experience report no operative mortality and two remote deaths [2]. Other series do not report long-term outcome to inform on long-term mortalilty [3]. Our own experience reports no operative deaths or long-term mortality at 2.8 years (0.4-13.8) of follow-up. We would agree that the literature and our experience suggest negligible operative mortality.

\section{References}

1. Guzman R, Lee M, Achrol A et al (2009) Clinical outcome after 450 revascularisation procedures for moyamoya disease. J Neurosurg 111:927-935

2. Scott RM, Smith RL, Robertson RL et al (2004) Long-term outcome in children with moyamoya syndrome after cranial revascularisation by pial synangiosis. J Neurosurg 100(2 Suppl Pediatrics): $142-149$

3. Ibrahimi DM, Tamargo RJ, Ahn ES (2010) Moyamoya disease in children. Childs Nerv Syst 26:1297-1308

J. Ng $(\bowtie) \cdot$ V. Ganesan

Neurology Department,

Great Ormond Street Hospital for Children NHS Trust,

London, UK

e-mail: drjoanneng@doctors.org.uk

D. Thompson · J. P. S. Lumley

Neurosurgery Department,

Great Ormond Street Hospital for Children,

London, UK

D. E. Saunders

Radiology Department,

Great Ormond Street Hospital for Children,

London, UK

V. Ganesan

Neurosciences Unit, UCL Institute of Child Health,

London, UK 